\title{
Increased Expression of MMP17 Predicts Poor Clinical Outcomes in Epithelial Ovarian Cancer Patients
}

Chao Xiao

Zigong First People's Hospital

Yao Wang

Zigong First People's Hospital

Chengjian Cao ( $\nabla$ cccjj2011@163.com )

Zigong First People's Hospital

\section{Research Article}

Keywords: Ovarian cancer, matrix metalloproteinases, prognosis, Kaplan-Meier plotter

Posted Date: November 3rd, 2021

DOl: https://doi.org/10.21203/rs.3.rs-1034397/v1

License: (a) (i) This work is licensed under a Creative Commons Attribution 4.0 International License. Read Full License 


\section{Abstract}

Objective Ovarian cancer has the highest fatality rate among female reproductive system cancers, which is due to lack of biomarkers for prognosis. We aimed to evaluate the role of MMP17 in ovarian cancer tumorigenesis and prognosis.

Methods Based on the epithelial ovarian cancer (EOC) on The Cancer Genome Atlas (TCGA) database, we determined the expression of MMP17 using the Wilcoxon rank sum test. The biological functions of MMP17 were evaluated using the Metascape database and Gene Set Enrichment Analysis (GSEA). The association between MMP17 and immune cell infiltration was investigated by single sample GSEA. Logistic analysis was applied to study the correlation between MMP17expression and clinicopathological characteristics. Finally, Cox regression analysis, Kaplan-Meier analysis, and nomograms were used to determine the predictive value of MMP17 on clinical outcomes in EOC patients.

Results MMP17 was much higher in EOC patients than in pericarcinous tissues $(P<0.001)$. MMP17associated DEGs were significantly enriched in cell extracellular matrix (ECM) degrading and corresponding pathways in the high MMP17 expression phenotype. MMP17 has a high sensitivity and specificity for EOC diagnosis, with an area under the curve (AUC) of 0.988. MMP17 expression was found to be an independent risk factor for overall survival [Hazard Ratio(HR): 1.488, P 0.001], progression-free interval (HR: 1.347, P 0.01 ), and disease-specific survival (HR: 1.548, P 0.01).

Conclusion Increased MMP17 expression in EOC may contribute to carcinogenesis by degrading ECM and provide diagnostic and prognostic value for clinical outcomes.

\section{Background}

Ovarian cancer is a life-threatening malignant tumor for women all over the world, with the third highest incidence and the highest fatality rate among female reproductive system cancers[1]. The five-year survival rate for advanced ovarian cancer is between $30 \%$ and $40 \%[2]$. Advanced diagnosis and platinum resistance are responsible for the poor prognosis of ovarian cancer. Epithelial ovarian cancer(EOC) is the most prevalent histological subtype of ovarian cancer, accounting for 90 percent or more of all cases, and it has distinct genetic features that can help enhance the accuracy and efficacy of treatment $[3,4]$. This makes the discovery of novel biomarkers for early detection and prediction of ovarian cancer invasion and metastasis extremely helpful.

The destruction of extracellular matrix(ECM) is required for malignant invasion and metastasis.Matrix metalloproteinases(MMPs) are a huge family of calcium-dependent zinc-containing endopeptidases that may breakdown practically any extracellular matrix to accelerate tumor invasion and metastasis.[5-7]. Upregulation of MMP2, MMP9, and MMP14 has been linked to an increased risk of ovarian cancer.[7, 8]. MMP17 is a membrane-type specific MMP that interacts with MMP2 to degrade ECM. However, MMP17's potential involvement and underlying mechanism in EOC are still unknown. 
Using RNA sequencing and clinical data of EOC patients retrieved from The Cancer Genome Atlas (TCGA) database, we performed a bioinformatics analysis to determine the role of MMP17 in EOC carcinogenesis and prognosis. MMP-17 was found to be overexpressed in EOC, and its involvement in carcinogenesis was studied. Following that, we conducted a correlation analysis between MMP17 and a number of clinicopathological factors. Finally, we determined MMP-17's diagnostic and prognostic values. This research sheds new light on the mechanisms behind EOC carcinogenesis and identifies MMP17 as a prospective diagnostic and prognostic biomarker in EOC.

\section{Results}

\section{Clinical characteristics}

TCGA provided the clinical histories of 379 patients, including age, race, Federation International of Gynecology and Obstetrics(FIGO) stage, primary therapeutic outcome, histologic grade, venous invasion, lymphatic invasion, and tumor residual, as well as Overall survival(OS), disease-specific survival(DSS), and progression-free interval(PFI) events. The details ware presented in Table 1.

\section{Table 1}

Clinical Characteristics of The EOC Patients Based on TCGA 


\begin{tabular}{|c|c|c|c|}
\hline Characteristic & Level & $\begin{array}{l}\text { Low expression of } \\
\text { MMP17(n=189) }\end{array}$ & $\begin{array}{l}\text { High expression of } \\
\text { MMP17(n=190) }\end{array}$ \\
\hline \multirow[t]{4}{*}{ FIGO stage, n (\%) } & Stage I & $1(0.3 \%)$ & $0(0 \%)$ \\
\hline & Stage II & $11(2.9 \%)$ & $12(3.2 \%)$ \\
\hline & Stage III & $145(38.6 \%)$ & $150(39.9 \%)$ \\
\hline & Stage IV & $31(8.2 \%)$ & $26(6.9 \%)$ \\
\hline \multirow{4}{*}{$\begin{array}{l}\text { Primary therapy } \\
\text { outcome, n (\%) }\end{array}$} & PD & $7(2.3 \%)$ & $20(6.5 \%)$ \\
\hline & SD & $11(3.6 \%)$ & $11(3.6 \%)$ \\
\hline & PR & $15(4.9 \%)$ & $28(9.1 \%)$ \\
\hline & $\mathrm{CR}$ & $125(40.6 \%)$ & $91(29.5 \%)$ \\
\hline \multirow[t]{3}{*}{ Race, n (\%) } & Asian & $4(1.1 \%)$ & $8(2.2 \%)$ \\
\hline & $\begin{array}{l}\text { Black or African } \\
\text { American }\end{array}$ & $14(3.8 \%)$ & $11(3 \%)$ \\
\hline & White & $166(45.5 \%)$ & $162(44.4 \%)$ \\
\hline \multirow[t]{2}{*}{ Age, $n(\%)^{*}$} & $<=60$ & $113(29.8 \%)$ & $95(25.1 \%)$ \\
\hline & $>60$ & $76(20.1 \%)$ & $95(25.1 \%)$ \\
\hline \multirow[t]{4}{*}{ Histologic grade, n (\%) } & $\mathrm{G} 1$ & $0(0 \%)$ & $1(0.3 \%)$ \\
\hline & $\mathrm{G} 2$ & $22(6 \%)$ & $23(6.2 \%)$ \\
\hline & G3 & $160(43.4 \%)$ & $162(43.9 \%)$ \\
\hline & G4 & $1(0.3 \%)$ & $0(0 \%)$ \\
\hline \multirow[t]{2}{*}{ Venous invasion, n (\%) } & No & $23(21.9 \%)$ & $18(17.1 \%)$ \\
\hline & Yes & $29(27.6 \%)$ & $35(33.3 \%)$ \\
\hline \multirow{2}{*}{$\begin{array}{l}\text { Lymphatic invasion, } \mathrm{n} \\
(\%)\end{array}$} & No & $28(18.8 \%)$ & $20(13.4 \%)$ \\
\hline & Yes & 49 (32.9\%) & $52(34.9 \%)$ \\
\hline \multirow[t]{2}{*}{ Tumor residual, n (\%) } & NRD & $32(9.6 \%)$ & $35(10.4 \%)$ \\
\hline & $\mathrm{RD}$ & $131(39.1 \%)$ & $137(40.9 \%)$ \\
\hline \multirow[t]{2}{*}{ OS event, n (\%) } & Alive & $79(20.8 \%)$ & $68(17.9 \%)$ \\
\hline & Dead & $110(29 \%)$ & $122(32.2 \%)$ \\
\hline \multirow[t]{2}{*}{ DSS event, n (\%) } & Alive & $83(23.4 \%)$ & 71 (20.1\%) \\
\hline & Dead & 94 (26.6\%) & 106 (29.9\%) \\
\hline
\end{tabular}




\begin{tabular}{|c|c|c|c|}
\hline Characteristic & Level & $\begin{array}{l}\text { Low expression of } \\
\text { MMP17(n=189) }\end{array}$ & $\begin{array}{l}\text { High expression c } \\
\text { MMP17 }(n=190)\end{array}$ \\
\hline \multirow[t]{2}{*}{ PFI event, n (\%) } & Alive & $50(13.2 \%)$ & $52(13.7 \%)$ \\
\hline & Dead & $139(36.7 \%)$ & $138(36.4 \%)$ \\
\hline Age, meidan (IQR) & & $58(50,66)$ & $60.5(51,71)$ \\
\hline
\end{tabular}

Using the TCGA database, MMP17 was found to be significantly over expressed in 13 of the 33 cancer types investigated, as shown in Figure 1A. MMP17 expression was much higher in EOC patients than in pericarcinous tissues $(P<0.001$, Figure 1B).

Based on median MMP17 expression in EOC, 427 OC patients were split into two groups: high- and lowMMP17 expression groups. Following that, we looked at the mRNA expression of two separate groups. Volcano plots (Figure 1C) indicated 753 mRNAs (641 upregulated and 112 downregulated) that were recognized as DEGs (absolute value of fold change $>1.0, P \llbracket 0.05$ ). In addition, a heatmap was employed to depict representative DEGs (Figure 1D).

\section{Functional annotation of MMP17 Associated differentially expressed genes in EOC}

Several EOC-related pathways were enhanced, as shown in Figures 2A-C, including extracellular matrix organization (GO:0030198), skeletal system development (G0:0001501), blood vessel development (GO:0001568), and cell substrate adhesion (GO:0001568) (GO: 0031589).Furthermore, MMP17-associated DEGs were significantly enriched in cell extracellular matrix (ECM) clusters (Figures $3 \mathrm{~A}-\mathrm{F}$ ), including extracellular matrix organization [normalized enrichment score $(N E S)=2.889$, adjusted $P=0.011$ ], $E C M$ receptor interaction (NES $=2.797$, adjusted $P=0.011)$, ECM degradation $(N E S=2.752$, adjusted $P=0.011)$, EMC proteoglycans $(\mathrm{NES}=2.691$, adjusted $\mathrm{P}=0.011)$, EMC glycoproteins $(\mathrm{NES}=2.683$, adjusted $\mathrm{P}=0.011)$ and collagen degradation(NES $=2.675$, adjusted $\mathrm{P}=0.011)$. We also constructed a PPI network to illustrate the MMP17 served as the hub gene related to another ten genes(Figure 4).

\section{Association of MMP17 and immune cell infiltration in EOC}

The SSGSEA method was used to determine the infiltration of 24 immune cell types in EOC, and then Spearman's analysis was used to evaluate the relationship between MMP17 and immune cell infiltration. $\operatorname{Tem}(R=0.357, P 0.001)$, macrophages $(R=0.333, P 0.001)$, and NK cells $(R=0.389, P 0.001)$ were all 
strongly linked with MMP17 expression, as shown in Figure 5A. Th17 cells $(R=-0.195, P 0.001)$ and NK CD56bright cells $(R=-0.107, P=0.037)$ were found to have a negative relationship with MMP17. We assessed at the levels of infiltration of the most important immune cells (Figure 5B,C) in different MMP17 groups, and the results were consistent with those in Figure 5A.

\section{Predictive value of MMP17 for EOC diagnosis and prognosis}

The evaluation of MMP17 on discriminating EOC diagnosis was demonstrated using a ROC curve. MMP17 has a high sensitivity and specificity for EOC diagnosis, with an area under the curve (AUC) of 0.988. (Figure $6 \mathrm{~A})$. Following that, K-M analyses were used to confirm MMP17's prediction of clinical outcomes. Overall survival (HR: 1.69, P 0.001), progression-free interval (HR: 1.42, $P=0.004)$, and disease-specific survival (HR: $1.72, \mathrm{P} 0.001$ ) for high-MMP17 exprssion groups were all substantially worse than for low-MMP17 groups, as illustrated in Figures 6B-D.

We also ran a multivariate Cox regression analysis to see if MMP17 had any predictive value for clinical outcomes. In multivariate Cox regression, MMP17 expression was found to be an independent risk factor for overall survival (HR: 1.488, P 0.001), progression-free interval (HR: 1.347, P 0.01), and disease-specific survival (HR: 1.548, P 0.01), however, FIGO stage, age, and race showed no prognostic advantages for clinical outcomes,As shown in Table 2.

Each multivariate Cox regression analysis' statistically significant prognostic factors were then utilized to design a prognostic nomogram, and a calibration curve was constructed to assess the nomogram's effectiveness. FIGO stage, age, and race, as well as MMP17, were included into the nomogram to predict OS(C-index of 0.694 )(Figure 7A)], DSS(C-index of 0.701 )(Figure 7C)], and PFI(C-index of 0.653 )(Figure 7E)].The calibration curves for the three nomograms for 1-, 3-, and 5-year clinical outcomes all showed promising results, with the exception of the 3- and 5-year predictions for DSS and forecast PFI, which were lacking in data (Figures 7B,D,F) 


\section{TABLE 2}

Cox regression analysis for clinical outcomes in EOC patients.

\begin{tabular}{|c|c|c|c|c|c|c|}
\hline \multirow[t]{2}{*}{ Characteristics } & \multicolumn{2}{|c|}{$\begin{array}{l}\text { HR for overall survival } \\
(95 \% \mathrm{Cl})\end{array}$} & \multicolumn{2}{|c|}{$\begin{array}{l}\text { HR for progression-free } \\
\text { interval (95\% Cl) }\end{array}$} & \multicolumn{2}{|c|}{$\begin{array}{l}\text { HR for disease-specific } \\
\text { survival ( } 95 \% \mathrm{Cl})\end{array}$} \\
\hline & Univariate & Multivariate & Univariate & Multivariate & Univariate & Multivariate \\
\hline $\begin{array}{l}\text { Age (>60 vs. } \\
\leq 60 \text { years) }\end{array}$ & $1.355^{\star}$ & 1.316 & 1.255 & NA & 1.255 & NA \\
\hline $\begin{array}{l}\text { FIGO stage }(I \& \| l \\
\text { vs. III\&IV) }\end{array}$ & 2.115 & 2.602 & 1.573 & 1.558 & 2.276 & 2.474 \\
\hline $\begin{array}{l}\text { Primary } \\
\text { therapy } \\
\text { outcome } \\
\text { (PD\&SD vs. } \\
\text { PR\&CR) }\end{array}$ & $0.301^{\star \star \star}$ & 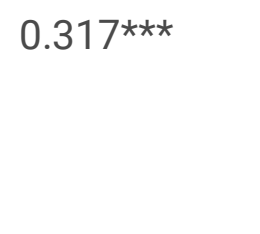 & $0.457 * \star \star$ & $0.507^{\star \star \star}$ & $0.294^{\star \star \star}$ & $0.33^{* * *}$ \\
\hline $\begin{array}{l}\text { MMP17(high } \\
\text { vs. low) }\end{array}$ & $1.639 * \star \star$ & $1.488 * \star \star$ & $1.39 * *$ & $1.347^{\star \star}$ & $1.693^{\star \star \star}$ & $1.548 \star \star$ \\
\hline $\begin{array}{l}\text { Race (White } \\
\text { vs. Black } \\
\text { or African } \\
\text { American and } \\
\text { Asian) }\end{array}$ & 0.637 & 0.61 & 0.843 & NA & $0.592^{*}$ & 0.601 \\
\hline
\end{tabular}

HR, hazard ratio; EOC, Epithelial Ovarian Caner, $\mathrm{Cl}$, confidence interval. ${ }^{*} \mathrm{P}<0.05$; $* \star \mathrm{P}<0.01$; ***P< 0.001 .

\section{Prognostic performance of MMP17 in EOC subgroups}

The next step was to see if MMP17 had any prognostic value for clinical outcomes in a variety of clinicopathological categories. In certain subgroups, we ran Cox regression analysis, and the results were shown as forest plots (Figure 8). In patients with tumor (HR = 1.76, $P=0.000)$, FIGO stage III-IV $(H R=1.72, P$ $=0.000)$, histology G3-4 $(H R=1.77, P=0.000)$, and tumor residual $(H R=1.60, P=0.001), M M P 17$ was a significant risk factor for overall survival(Figure 8A). Figure 8B shows similar findings for disease-specific survival and progression-free intervals (Figure $8 \mathrm{C}$ ). MMP17 was also found to be an independent risk factor for all ages and anatomic subdivisions.

In addition, we reported K-M analyses for clinical outcomes (overall survival, progression-free interval, and disease-specific survival) in four representative subgroups: age under 60 , tumor status, and anatomic neoplasm subdivision (Figure 9). All of these findings showed that the low-MMP17 expression groups had considerably superior clinical outcomes.

\section{Discussion}


In our study, we found that MMP17 was significantly elevated in gynecologic cancers, such as UCEC(Uterine Corpus Endometrial Carcinoma \and CESC(Cervical squamous cell carcinoma and endocervical adenocarcinoma). Moreover, MMP17 was also upregulated in tumors, such as THCA(Thyroid carcinoma), PAAD (Pancreatic adenocarcinoma),PRAD (Prostate adenocarcinoma),READ(Rectum adenocarcinoma), SKCM(Skin Cutaneous Melanoma), and other systems. As a result, MMP17 might be an important hub gene in carcinogenesis.

In EOC patients, we discovered a strong link between MMP17 expression and age, histologic grade, and clinical stage. MMP17 was also found to be an independent risk factor for all ages and anatomic divisions. Finally, we proved MMP17's prognostic value for overall survival, progression-free interval, and diseasespecific survival in EOC patients, particularly those with advanced stages, high histologic grades, and residual tumor.

By examining information from the TCGA-OV, we focused on expression patterns, clinicopathological correlations, and the clinical importance of an extracellular matrix (ECM) protease, MMP17, in EOC. MMP17 expression was shown to be significantly higher in EOC. DEGs associated with increased MMP17 expression were found to be concentrated in cell ECM pathways.

Further, we attempted to describe the potential functions and mechanisms involving MMP17 in EOC. The initial barrier in the process of tumor metastasis was the ECM and substrate, and its degradation was the critical link in tumor invasion and metastasis. MMPs degrading activities in ECM cause changes in its structure and expression of its cellular surface receptors, resulting in the occurrence, development, invasion, and metastasis of malignant tumors. $[6,7,9]$.

Embryonic development, wound healing, blood vessel development, and skeletal system development were all linked to MMP17 expression, according to functional annotation of MMP17-associated DEGs. Several cell ECM related pathways were enriched in the high-MMP17 group, according to supplementary GSEA. MMP17 could trigger MMP2 to improve type IV collagen and gelatin cleavage and denaturation. MMP17 operates as a major ECM regulator in EOC, according to the results presented above.

In addition, we discovered a link between MMP17 expression and immune cell infiltration. MMP17 expression was associated with cytotoxic cells, Th17 cells, and NK CD56bright cells in a negative way. Th17 cells activate effector CD8(+) T cells and induce anticancer immune responses by recruiting immune cells into tumors[10]. Antitumor immunity can be initiated by cytotoxic CD8+ T cells, and NK CD56bright cells have been shown to have antitumor responses after being primed with IL-15[11]. As a result, overexpressed MMP17 appeared to wreak havoc on tumor immunity, aid cancer cells in evading elimination, and hasten carcinogenesis. On the other hand, we discovered a substantial positive relationship between MMP17 expression and macrophage infiltration, which is linked to a poor prognosis in solid tumors and is linked to chemotherapy resistance in most cancers[11-13]. By boosting angiogenesis, enhancing migration and invasion, and decreasing antitumour immunity, macrophages may promote cancer development and progression. 
MMP17's clinical relevance in EOC is undeniable. Firstly, the AUC of the ROC curve for MMP17 discrimination of EOC diagnosis was 0.988 , indicating that MMP17 was a reliable biomarker for EOC diagnosis. Secondly, patients with increased MMP17 levels had a significantly reduced rate of overall survival, progression-free interval, and disease-specific survival. Lastly, MMP17's predictive value appeared to be stronger in patients with certain characteristics, such as advanced stage, high histologic grade, and residual tumor. All three qualities are, coincidentally, challenges in the therapeutic treatment of ovarian cancer. The predictive ability of MMP17 indicated a possibility that it could serve as a common prognostic biomarker for poor survival in EOC.

In a focusing on MPPs family study, reporting MMP17 was not associated with overall survival in ovarian cancer patient[14]. This dramatic result may related the database they took[15].

Despite the fact that we discovered a possible mechanism for MMP17 activity in EOC carcinogenesis and its predictive usefulness in clinical outcomes, our research had significant flaws. First, we were unable to assess a specific role for MMP17 in EOC treatment due to a lack of knowledge about treatments and their outcomes. Second, we primarily employed the TCGA database's RNA sequencing data, therefore we couldn't provide information on relative protein levels or MMP17's downstream pathways. As a result, more in vivo and in vitro research focusing on the direct mechanism of MMP17 activity in EOC will be needed.

\section{Conclusions}

Increased MMP17 expression in EOC may contribute to carcinogenesis by degrading ECM and provide diagnostic and prognostic value for clinical outcomes.

\section{Materials And Methods}

\section{Data processing and ethics statement}

The EOC in the TCGA database (https://portal.gdc.cancer.gov/) provided us with high-throughput sequencing RNA data [fragments per kilobase per million (FPKM) format] and corresponding clinicopathological information. A total of 379 EOC patients were included in the study. For this work, RNA sequencing data was converted from FPKM to TPM formats (transcripts per million reads). The TCGA database confirms that all written informed consents were received prior to data collection because it is open to the public under strict guidelines.

\section{Differentially expressed genes in EOC}

The 379 EOC patients were divided into two groups based on their MMP17 expression levels: high and low. A two-tailed hypothetical test based on negative binomial generalized linear models was used to identify differentially expressed genes (DEGs) between the two groups using the R package "DESeq2"[16], with thresholds of a log-fold change greater than 1.0 and an adjusted P-value less than 0.05 . To exhibit the results as heatmaps and volcano plots, the R package "ggplot2, edition 3.3.3" was used. 


\section{Functional annotation of MMP17 associated differentially expressed genes in EOC}

The DEGs were then processed on the Metascape database (https://metascape.org/) and online tool[17] for functional annotation. Analysis thresholds were established at minimum counts greater than 3 , enrichment factors greater than 1.5, and a P-value less than 0.01. The Gene Set Enrichment Analysis (GSEA)[18] of the DEGs in the two groups was also performed using the R package "clusterProfifiler" [19].As reference gene sets in GSEA, C2: curated gene sets from MSigDB collections were chosen.There were 626 clusters found in total, with clusters having a false discovery rate (FDR) of less than 0.25 and a P-value of less than 0.05 being considered significant.PPI networks were investigated using the STRING database (http://string$\mathrm{db}$. org)[20] and displayed using the Cytoscape software (version 3.9.0). [21].

\section{Association of MMP17 and immune cell infiltration in EOC}

Infiltration enrichment of 24 common immune cells[22] was shown using the single sample GSEA technique from the R package "GSVA version 1.34.0"[23] , as shown in figure 4A. Second, the relationship between MMP17 expression and immune cell infiltration was assessed using Spearman's analysis, and immune cell infiltration levels were compared using the Wilcoxon rank sum test for high and low MMP-17 expression groups.

\section{Clinical significance of MMP17 expression in EOC}

The predictive potential of MMP17 for EOC diagnosis was tested by comparing MMP17 expression in EOC and normal tissues using receiver operating characteristic (ROC) analysis. The EOC data was extracted from TCGA, as well as the corresponding normal tissue data from GTEx. The Toil procedure [24] unified the treatment of UCSC XENA (https://xenabrowser.net/datapages/) RNAseq data into TCGA and GTEx TPM format. The analysis was carried out using the R package "pROCversion1.17.0.1" and the visualization was carried out using "ggplot2 version 3.3.3."

A published study[25] provided information on EOC patients' clinical outcomes, including overall survival, progression-free interval, and disease-specific survival. For prognosis analysis, Kaplan-Meier (K-M) analysis, univariate, and multivariate Cox regression analysis were used. The nomograms and calibration plots were created using the R packages "rms version 6.2-0" and "survival version3.2-10." R (v3.6.3) was used to conduct all of the aforementioned statistical studies, with P-values less than 0.05 deemed significant.

\section{Abbreviations}

TCGA,The Cancer Genome Atlas; GSEA, Gene Set Enrichment Analysis; PD,progressive disease; SD,stable disease; $\mathrm{PR}$,partial response; $\mathrm{CR}$,complete response; $\mathrm{RD}$,radical resection;IQR, interquartile range; FIGO,Federation International of Gynecology and Obstetrics;HR,hazard ratio; OS,Overall survival; PFI, progression-free interval; DSS, disease-specific survival; NES, normalized enrichment score;UCEC,Uterine 
Corpus Endometrial Carcinoma; THCA,Thyroid carcinoma; PAAD,Pancreatic adenocarcinoma; PRAD,Prostate adenocarcinoma; READ,Rectum adenocarcinoma;SKCM,Skin Cutaneous Melanoma; CESC,Cervical squamous cell carcinoma and endocervical adenocarcinoma;DEGs, differentially expressed genes; $\mathrm{K}$ M,Kaplan-Meier;

\section{Declarations}

\section{Author contributions}

Chao Xiao and Yao Wang is the principle investigator. Chao Xiao and Yao Wang conducted statistical analysis and draft the manuscript. Chao Xiao and Chengjian Cao performed data management and bioinformatic analysis. Chao Xiao and Chengjian Cao edited and revised the manuscript. All authors read and approved the final manuscript.

\section{Competing interests}

The authors declare that they have no competing interests.

\section{Availability of data and materials}

Please contact author for data requests.

\section{Consent for publication}

TCGA database portal is publicly available databases, thus individual patient informed consent was not required.

\section{Ethics approval and consent to participate}

Not applicable

\section{Funding}

Not applicable

\section{Acknowledgement}


The authors gratefully acknowledge The Gene Cancer Genome Atlas (TCGA) database and the academic tool(www.xiantao.love邓.

\section{References}

1. Stewart C, Ralyea C, Lockwood S. Ovarian Cancer: An Integrated Review. Semin Oncol Nurs. 2019;35:151-6.

2. Chen W, Zheng R, Baade PD, Zhang S, Zeng H, Bray F, et al. Cancer statistics in China, 2015. CA Cancer J Clin. 2016;66:115-32.

3. Reid BM, Permuth JB, Sellers TA. Epidemiology of ovarian cancer: a review. Cancer Biol Med. 2017;14:932.

4. Lheureux S, Gourley C, Vergote I, Oza AM. Epithelial ovarian cancer. Lancet. 2019;393:1240-53.

5. Yokoi A, Yoshioka Y, Yamamoto Y, Ishikawa M, Ikeda SI, Kato T, et al. Malignant extracellular vesicles carrying MMP1 mRNA facilitate peritoneal dissemination in ovarian cancer. Nat Commun. 2017;8:14470.

6. Zhang Y, Chen Q. Relationship between matrix metalloproteinases and the occurrence and development of ovarian cancer. Braz J Med Biol Res. 2017;50:e6104.

7. Vos MC, van Tilborg A, Brands WJ, Boll D, van Hamont $D$, van der Putten $H$, et al. Polymorphisms in MMP-14 and MMP-2 genes and ovarian cancer survival. Cancer Biomark. 2019;25:233-41.

8. Wang X, Yang B, She Y, Ye Y. The IncRNA TP73-AS1 promotes ovarian cancer cell proliferation and metastasis via modulation of MMP2 and MMP9. J Cell Biochem. 2018;119:7790-9.

9. Bourboulia D, Stetler-Stevenson WG. Matrix metalloproteinases (MMPs) and tissue inhibitors of metalloproteinases (TIMPs): Positive and negative regulators in tumor cell adhesion. Semin Cancer Biol. 2010;20:161-8.

10. Guéry L, Hugues S. Th17 Cell Plasticity and Functions in Cancer Immunity. Biomed Res Int. 2015;2015:314620.

11. Wagner JA, Rosario M, Romee R, Berrien-Elliott MM, Schneider SE, Leong JW, et al. CD56bright NK cells exhibit potent antitumor responses following IL-15 priming. J Clin Invest. 2017;127:4042-58.

12. Cassetta L, Pollard JW. Targeting macrophages: therapeutic approaches in cancer. Nat Rev Drug Discov. 2018;17:887-904.

13. Xia Y, Rao L, Yao H, Wang Z, Ning P, Chen X. Engineering Macrophages for Cancer Immunotherapy and Drug Delivery. Adv Mater. 2020;32:e2002054.

14. Zeng L, Qian J, Zhu F, Wu F, Zhao H, Zhu H. The prognostic values of matrix metalloproteinases in ovarian cancer. J Int Med Res. 2020;48:300060519825983.

15. Lánczky A, Nagy Á, Bottai G, Munkácsy G, SzabóA, Santarpia L, et al. miRpower: a web-tool to validate survival-associated miRNAs utilizing expression data from 2178 breast cancer patients. Breast Cancer Res Treat. 2016;160:439-46.

16. Love MI, Huber W, Anders S. Moderated estimation of fold change and dispersion for RNA-seq data with DESeq2. GENOME BIOL. 2014;15:550. 
17. Zhou Y, Zhou B, Pache L, Chang M, Khodabakhshi AH, Tanaseichuk O, et al. Metascape provides a biologist-oriented resource for the analysis of systems-level datasets. Nat Commun. 2019;10:1523.

18. Subramanian A, Tamayo P, Mootha VK, Mukherjee S, Ebert BL, Gillette MA, et al. Gene set enrichment analysis: a knowledge-based approach for interpreting genome-wide expression profiles. Proc Natl Acad Sci U S A. 2005;102:15545-50.

19. Yu G, Wang LG, Han Y, He QY. clusterProfiler: an R package for comparing biological themes among gene clusters. OMICS. 2012;16:284-7.

20. Szklarczyk D, Gable AL, Lyon D, Junge A, Wyder S, Huerta-Cepas J, et al. STRING v11: protein-protein association networks with increased coverage, supporting functional discovery in genome-wide experimental datasets. Nucleic Acids Res. 2019;47:D607-607D613.

21. Shannon P, Markiel A, Ozier O, Baliga NS, Wang JT, Ramage D, et al. Cytoscape: a software environment for integrated models of biomolecular interaction networks. Genome Res. 2003;13:2498-504.

22. Bindea G, Mlecnik B, Tosolini M, Kirilovsky A, Waldner M, Obenauf AC, et al. Spatiotemporal dynamics of intratumoral immune cells reveal the immune landscape in human cancer. Immunity. 2013;39:78295.

23. Hänzelmann S, Castelo R, Guinney J. GSVA: gene set variation analysis for microarray and RNA-seq data. BMC Bioinformatics. 2013;14:7.

24. Vivian J, Rao AA, Nothaft FA, Ketchum C, Armstrong J, Novak A, et al. Toil enables reproducible, open source, big biomedical data analyses. Nat Biotechnol. 2017;35:314-6.

25. Liu J, Lichtenberg T, Hoadley KA, Poisson LM, Lazar AJ, Cherniack AD, et al. An Integrated TCGA PanCancer Clinical Data Resource to Drive High-Quality Survival Outcome Analytics. Cell. 2018;173:40016.e11.

\section{Figures}




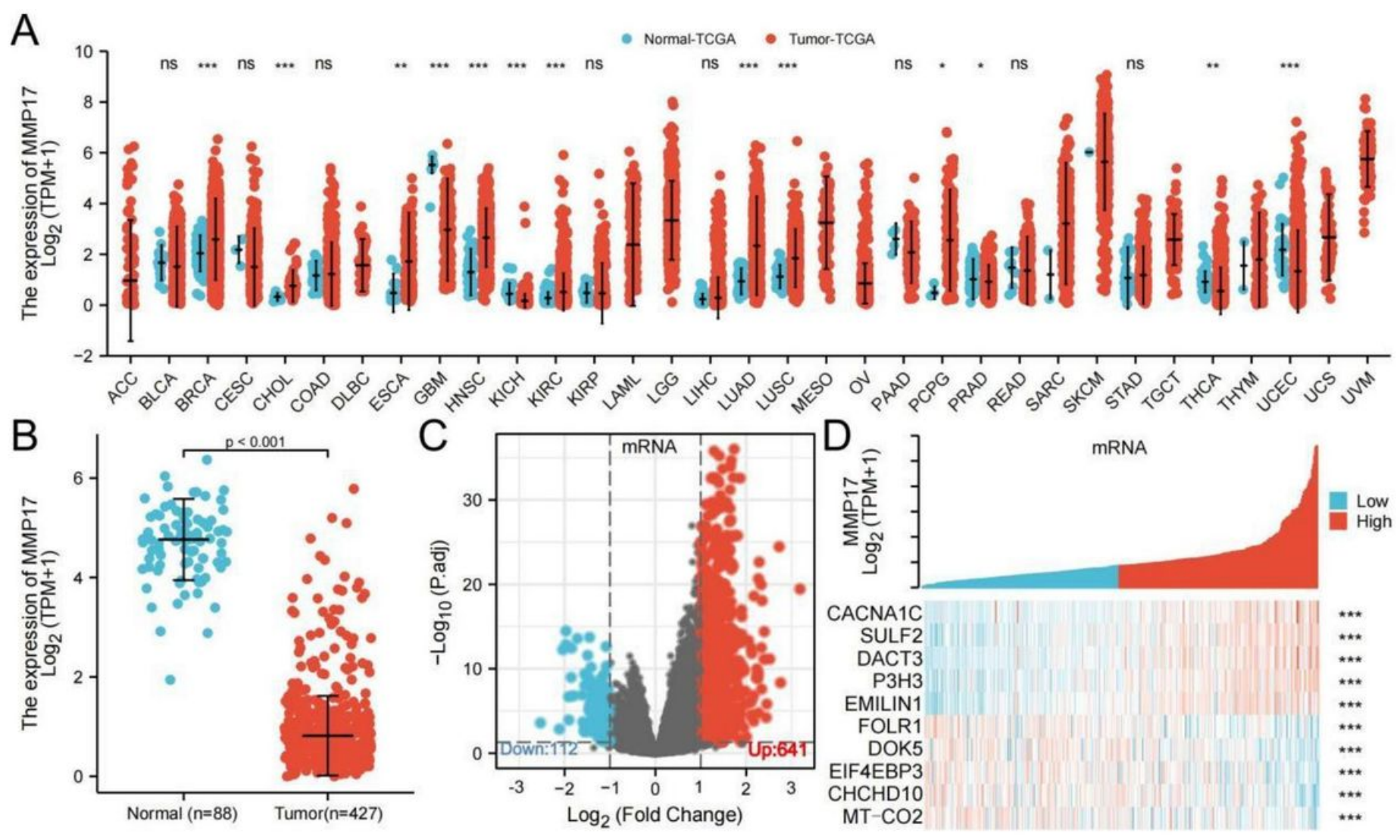

Figure 1

Differential mRNA expression profiles in EOC patients stratified by MMP17 levels. (A) The comparison of MMP17 expression between tumor and pericarcinous tissue in different types of cancers based on TCGA database. ns, $P \geq 0.05 ;{ }^{*}<<0.05 ;{ }^{*} P<0.01 ; * \star * P<0.001$. (B) MMP17 expression is higher in EOC than normal tissue. Shown are expression profiles of mRNA in two groups; and data are presented by volcano plots (C) and heatmaps (D). 


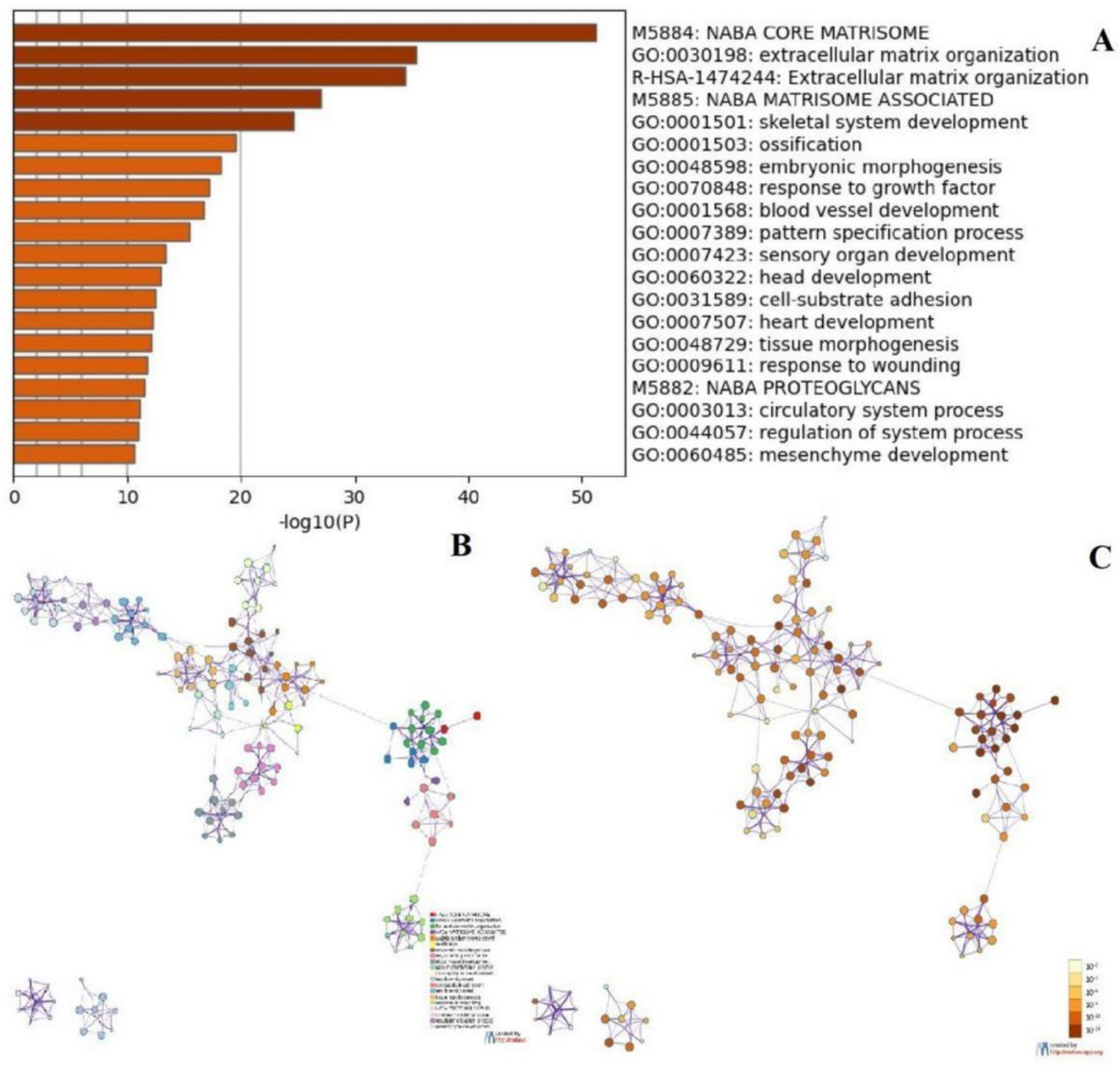

\section{Figure 2}

Functional annotation of differentially expressed genes (DEGs) in EOC with distinct MMP17 levels.

According to the Metascape database, 753 differentially expressed mRNAs between high- and low-MMP17 expression groups were used for functional annotation. All statistically enriched terms were identified and then hierarchically clustered into a tree (A) based on the threshold of kappa score as 0.3 . Representative terms from the cluster were converted into a network layout (B). The size of a node is proportional to the number of input genes that fall into that term, and the respective color represents its cluster identity. Terms with a similarity score $>0.3$ are linked by an edge (the thickness of the edge represents the similarity score). The same enrichment network presents nodes colored by the P-value (C). 
AEEACTOME_EXTRACELLULAR_MATRIX_ORGANRRROMIOME_DEGRADATION_OF_THE_EXTRACELLULAR_MATRIX
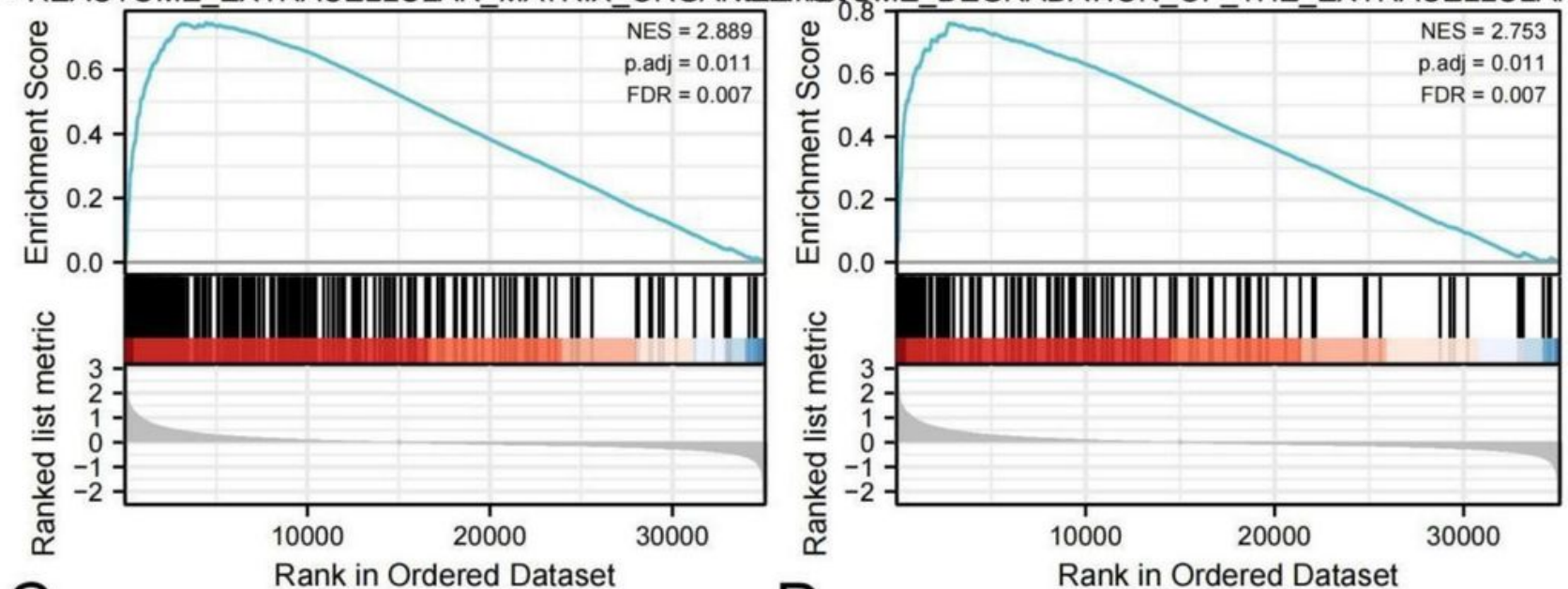

CEGG_ECM_RECEPTOR_INTERACTION
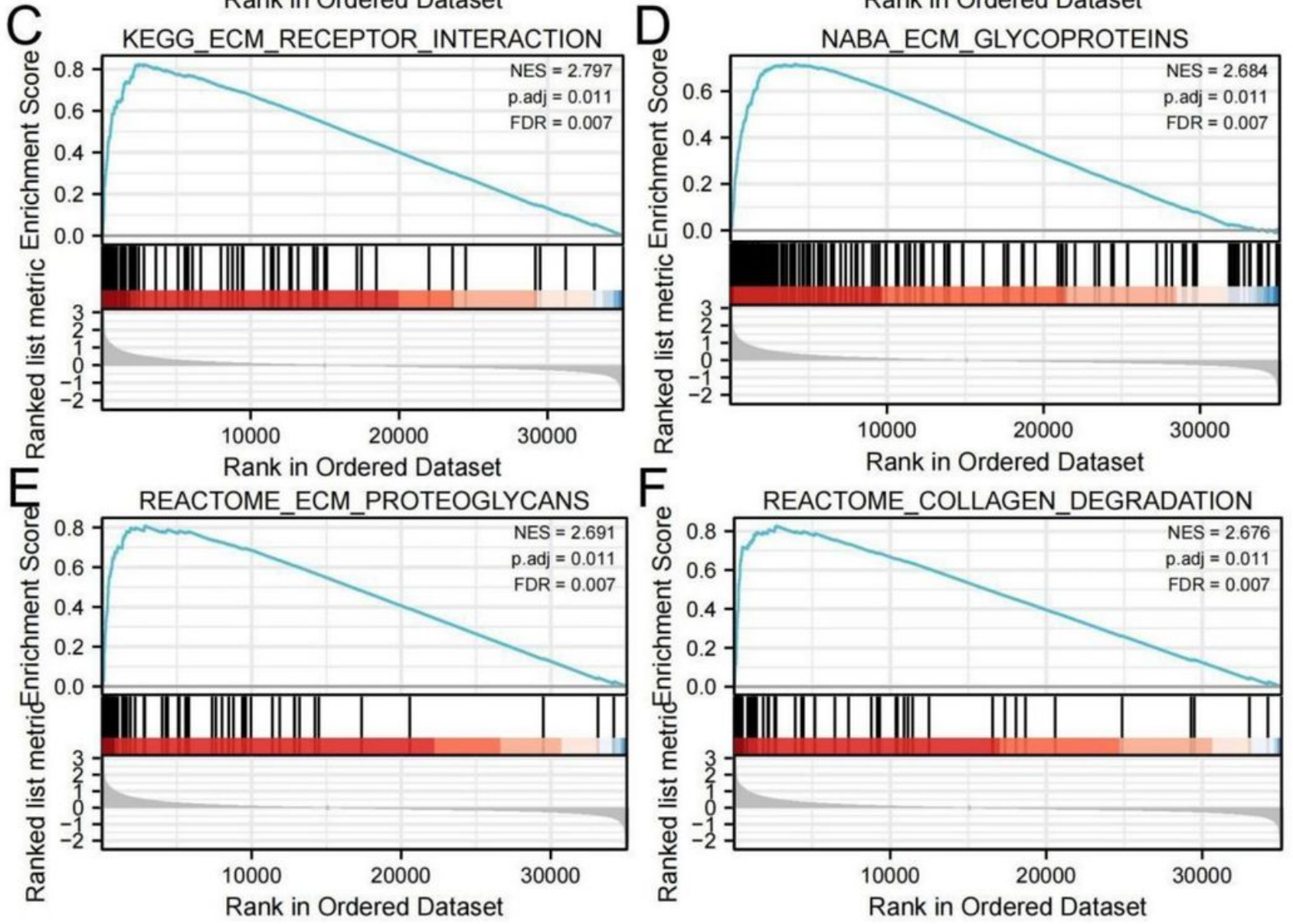

Figure 3

Representative Gene Set Enrichment Analysis of differentially expressed mRNAs between high- and lowMMP17 expression groups 


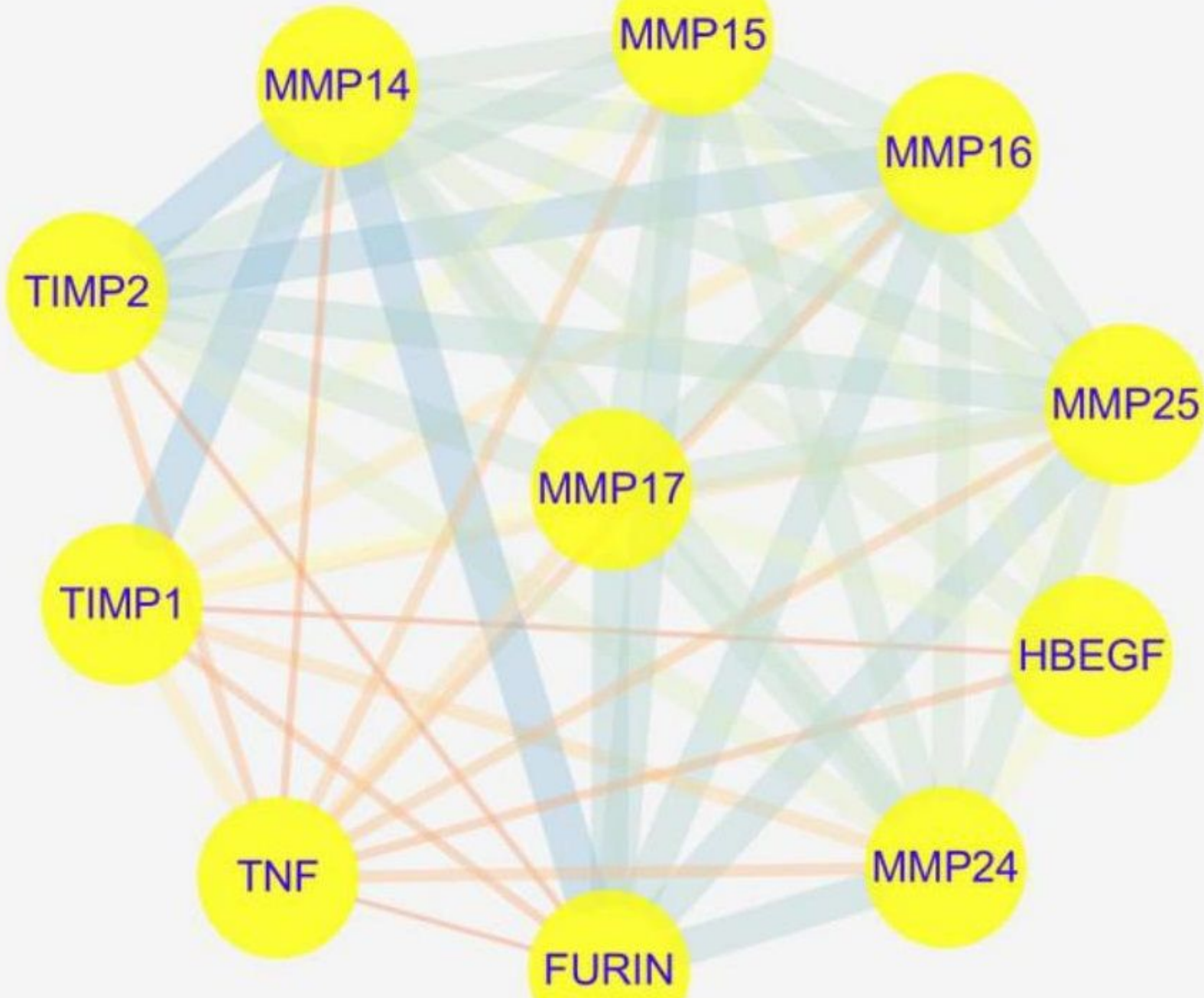

Figure 4

PPI network for MMP17, the most frequent 10 neighbor genes. 


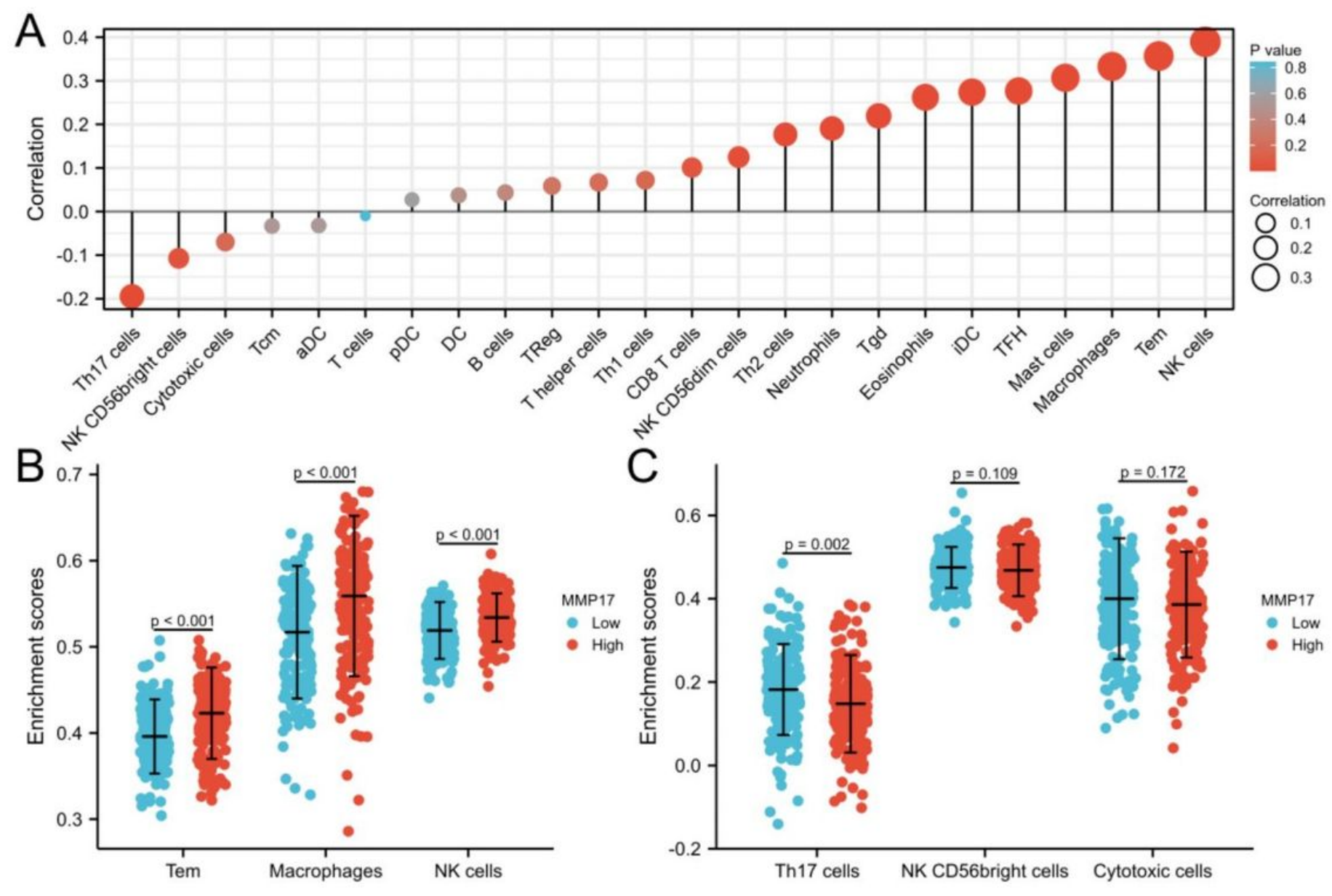

\section{Figure 5}

Correlation of immune cell infiltration and MMP17 expression in EOC patients. (A) Relationships among infiltration levels of 24 immune cell types and MMP17 expression profiles by Spearman's analysis. Shown is the comparison of infiltration levels of most correlated immune cells, including Tem, macrophages and NK cells (B), Th17 cells, NK CD56bright cells and Cytotoxic cells (C) between high- and low-MMP17 expression groups. aDC [activated DC]; B cells; CD8 T cells; Cytotoxic cells; DC; Eosinophils; iDC [immature DC];

Macrophages; Mast cells; Neutrophils; NK CD56bright cells; NK CD56dim cells; NK cells; pDC [Plasmacytoid DC]; T cells; T helper cells; Tcm [T central memory]; Tem [T effector memory]; Tfh [T follicular helper]; Tgd [T gamma delta]; Th1 cells; Th17 cells; Th2 cells; Treg. 


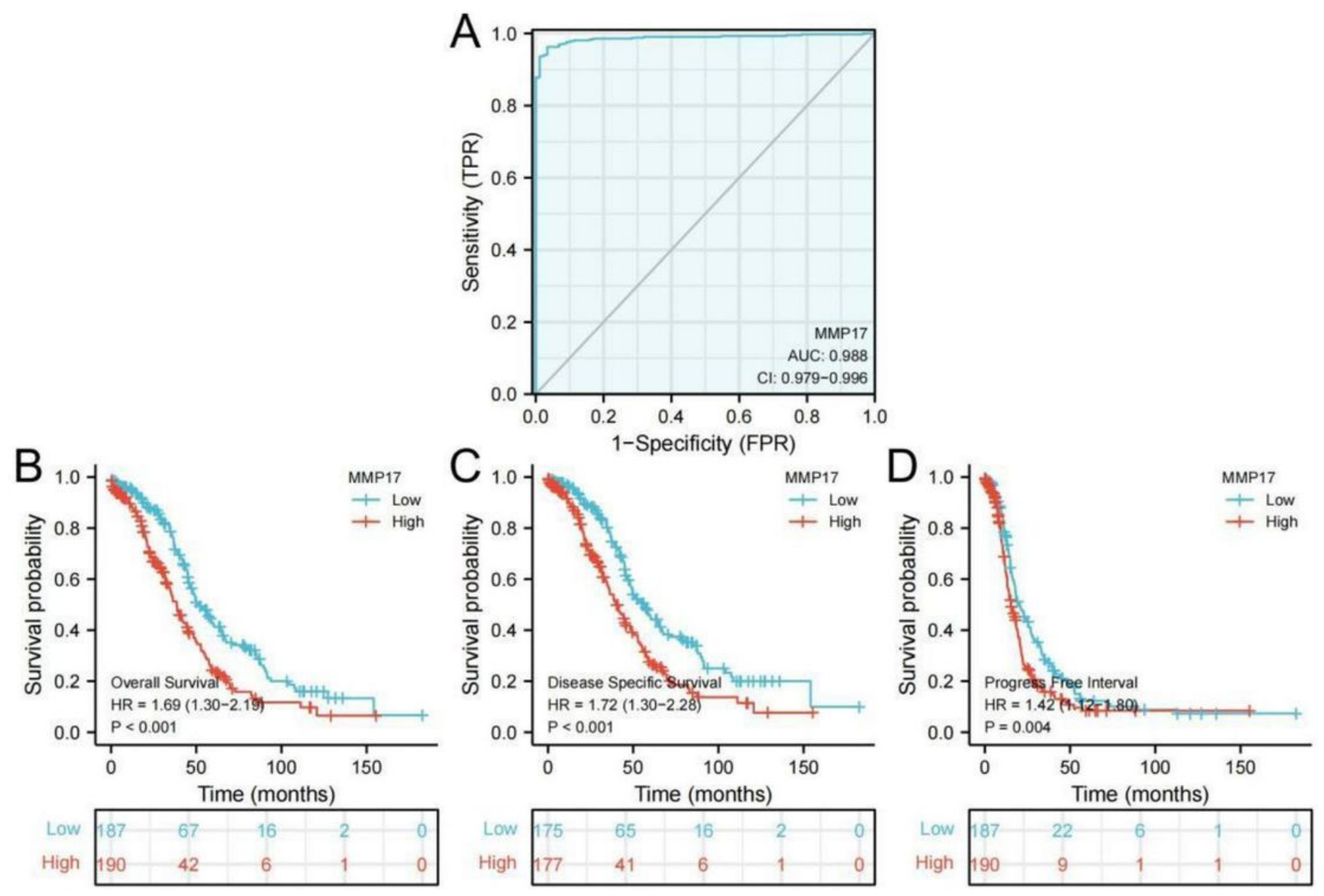

\section{Figure 6}

Predictive value of MMP17 expression for diagnosis and clinical outcomes in EOC patients. (A) Receiver operating characteristic (ROC) curve analysis evaluating the performance of MMP17 for EOC diagnosis. Shown are the Kaplan-Meier analyses comparing overall survival (B), progression-free interval (C), and disease-specific survival (D) between high- and low-MMP17 expression groups. 
A
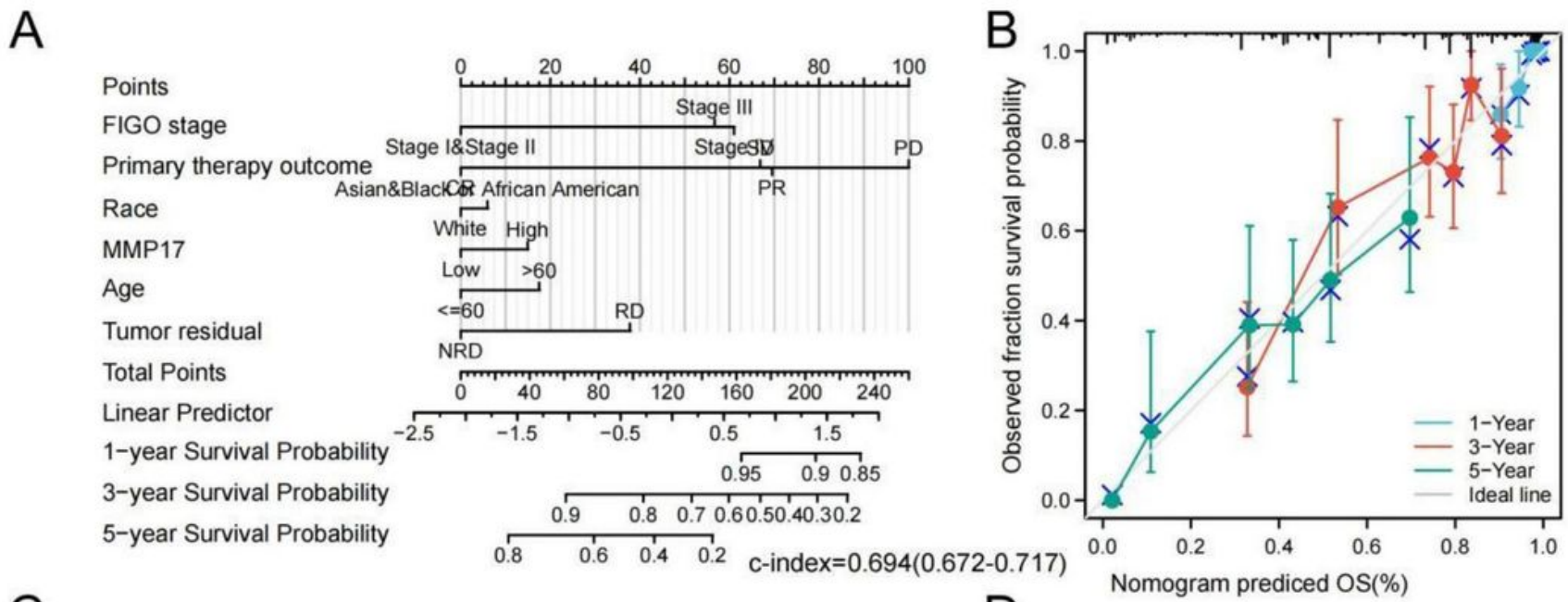

C
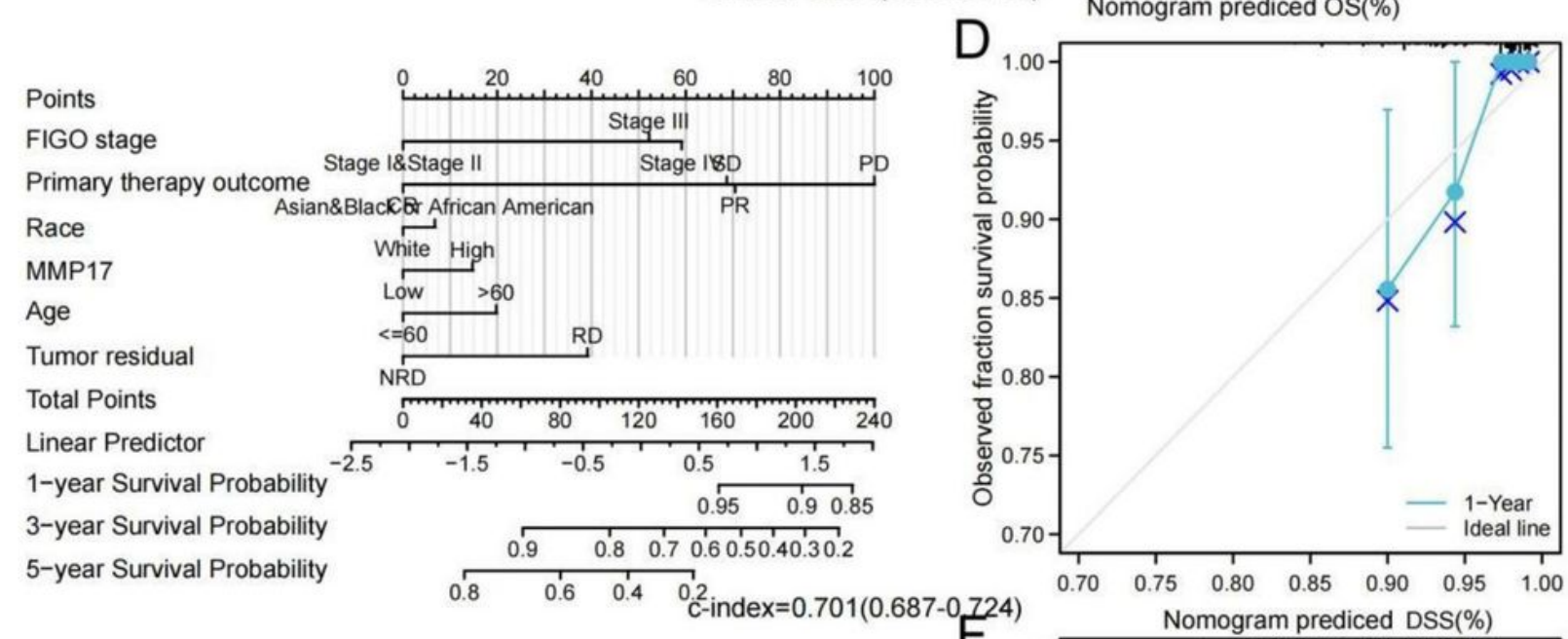

E
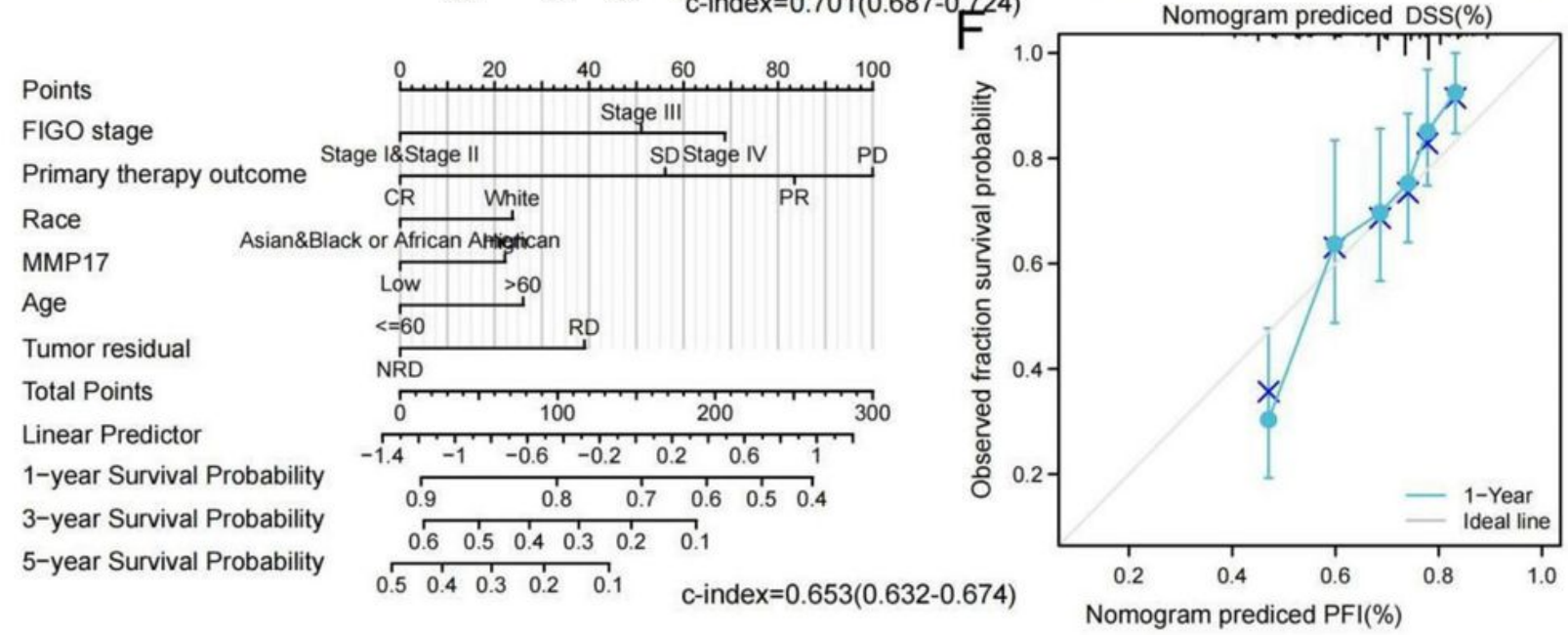

Figure 7

Construction and validation of nomograms based on MMP17 expression. Shown are the nomograms constructed to establish MMP17 expression-based risk scoring models for 1-, 3-, and 5-year overall survival (A), disease-specific survival (C), and progression-free interval(E). Calibration plots validating the efficiency of nomograms for overall survival (B), disease-specific survival(D), and progression-free interval(F). OS, overall survival; PFI, progression-free interval; DSS, disease-specific survival. 


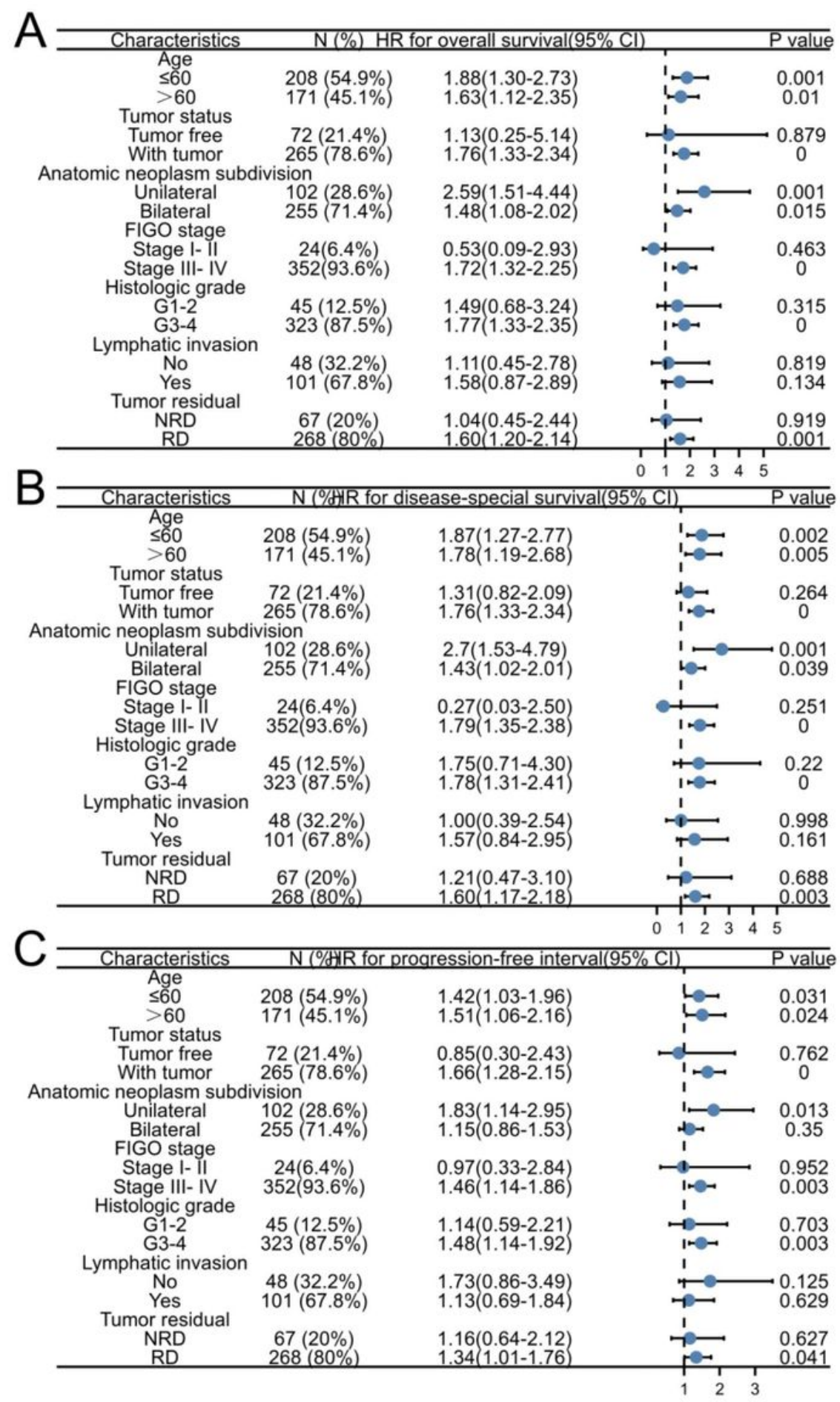

Figure 8

Prognostic performance of MMP17 on clinical outcomes in different EOC patient subgroups. Patients were divided into different subgroups according to age, tumor status, anatomic neoplasm subdivision, FIGO stage, histologic grade, lymphatic invasion and tumor residual. For each subgroup, the prognostic performance of MMP17 on overall survival (A), disease-specific survival(B), and progression-free interval(C) were evaluated by Cox regression, and the results are presented as hazard ratio. The bar represents the $95 \%$ confidence interval of hazard ratio, the diamond's size represents the significance of MMP17's performance. 

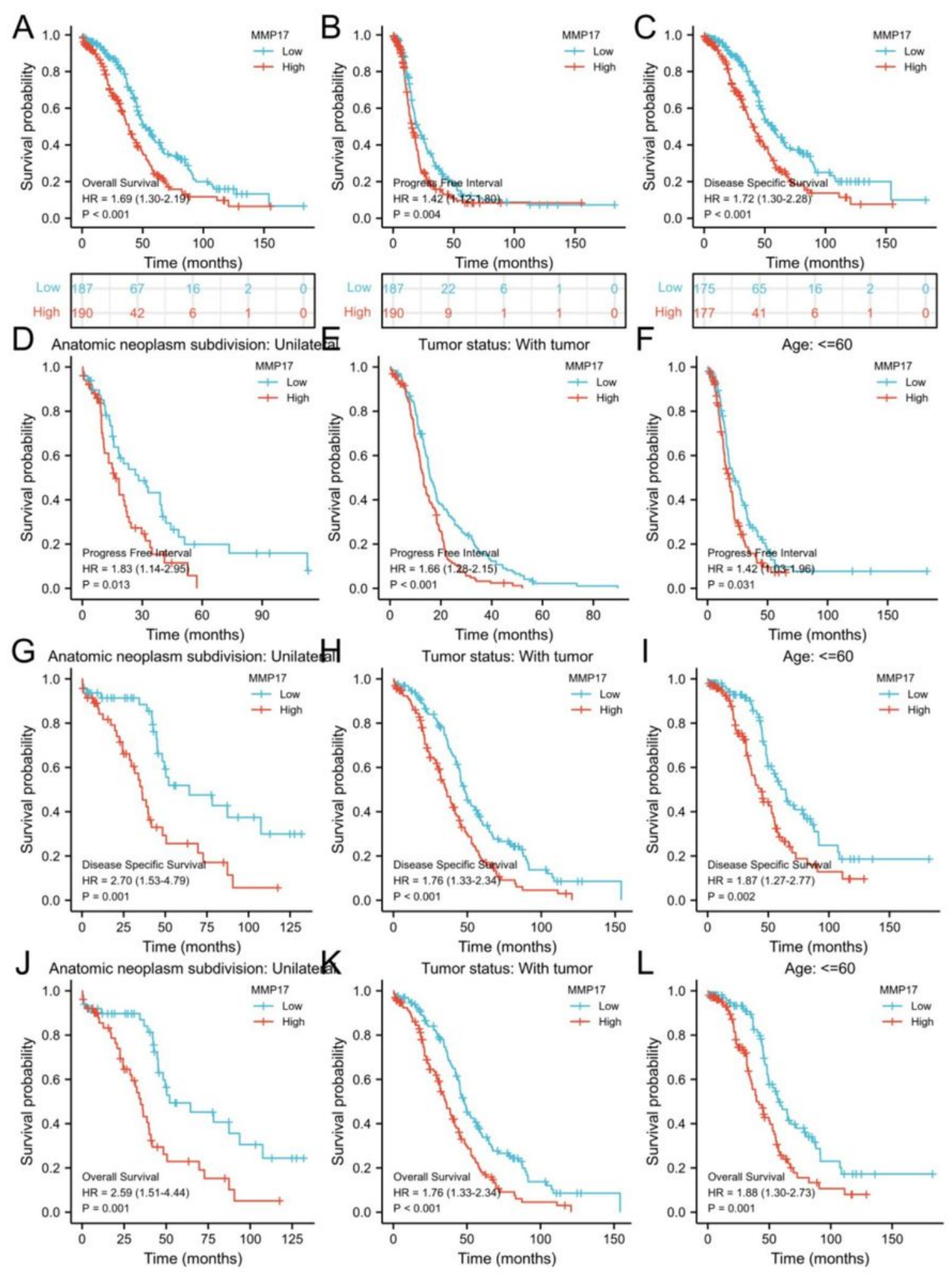

\section{Figure 9}

Distinct clinical outcomes based on MMP17 expression in EOC patient subgroups. Kaplan-Meier analysis showing the comparison of overall survival (A), progression-free interval (B), and disease-specific survival (C) between high- and low-MMP17 expression groups. The subgroups included age below 60 years $(F, I, L)$, tumor status $(\mathrm{E}, \mathrm{H}, \mathrm{K})$, and anatomic neoplasm subdivision( $\mathrm{D}, \mathrm{G}, \mathrm{J})$. 